\title{
Overweight and obesity in young Turkish, Moroccan and Surinamese migrants of the second generation in the Netherlands
}

\author{
Henriëtte Dijkshoorn ${ }^{1, *}$, Mary Nicolaou², Joanne K Ujcic-Voortman ${ }^{1}$, \\ Gea M Schouten ${ }^{3}$, Arianne J Bouwman-Notenboom ${ }^{4}$, Mary PH Berns ${ }^{5}$ and \\ Arnoud P Verhoeff ${ }^{1,6}$ \\ 'Department of Epidemiology, Documentation and Health Promotion, Public Health Service of Amsterdam, \\ PO Box 2200, 1000 CE Amsterdam, The Netherlands: ${ }^{2}$ Department of Social Medicine, Academic Medical \\ Centre, University of Amsterdam, Amsterdam, The Netherlands: ${ }^{3}$ Public Health Service of Rotterdam-Rijnmond, \\ Rotterdam, The Netherlands: ${ }^{4}$ Municipality of Utrecht, Public Health Service, Utrecht, The Netherlands: \\ ${ }^{5}$ Department of Epidemiology and Health Promotion, Public Health Service of The Hague, The Hague, \\ The Netherlands: 'Department of Sociology and Anthropology, University of Amsterdam, Amsterdam, \\ The Netherlands
}

Submitted 20 November 2012: Final revision received 24 June 2013: Accepted 11 July 2013: First published online 20 September 2013

\begin{abstract}
Objective: To examine differences in overweight and obesity of second-generation Turkish, Moroccan and Surinamese migrants $v$. first-generation migrants and the ethnic Dutch. We also studied the influence of sociodemographic factors on this association.

Design: Data were collected in 2008 in a cross-sectional postal and online health survey.

Setting: Four major Dutch cities.

Subjects: In the survey 42686 residents aged 16 years and over participated. Data from Dutch ( $n$ 3615) and second/first-generation Surinamese ( $n$ 230/139), Turkish ( $n$ 203/241) and Moroccan ( $n$ 172/187) participants aged 16-34 years were analysed using logistic regression with overweight (BMI $\geq 25 \cdot 0 \mathrm{~kg} / \mathrm{m}^{2}$ ) and obesity $\left(\mathrm{BMI} \geq 30 \cdot 0 \mathrm{~kg} / \mathrm{m}^{2}\right.$ ) as dependent variables. BMI was calculated from self-reported body height and weight. Sociodemographic variables included sex, age, marital status, educational level, employment status and financial situation. Results: After controlling for age, overweight (including obesity) was more prevalent in most second-generation migrant subgroups compared with the Dutch population, except for Moroccan men. Obesity rates among second-generation migrant men were similar to those among the Dutch. Second-generation migrant women were more often obese than Dutch women. Ethnic differences were partly explained by the lower educational level of second-generation migrants. Differences in overweight between second- and first-generation migrants were only found among Moroccan and Surinamese men.

Conclusions: We did not find a converging trend for the overweight and obesity prevalence from second-generation migrants towards the Dutch host population. Therefore, preventive interventions should also focus on second-generation migrants to stop the obesity epidemic.
\end{abstract}

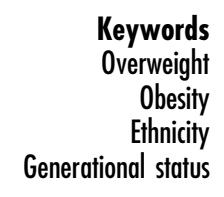

Keywords

verweight

Ethnicity

Generational status
Overweight (including obesity) is a major problem for public health. It is associated with many chronic diseases, such as CVD and diabetes mellitus ${ }^{(1)}$. Since 1980 the prevalence of overweight had increased worldwide, also in the Netherlands ${ }^{(2)}$. Migration to a Western culture seems to enlarge the risk of overweight ${ }^{(3,4)}$. Migrants from non-Western countries form an increasing proportion of the populations of many European countries. In the Netherlands, the major non-Western migrant groups originate from
Turkey, Morocco and Surinam (each $2 \%$ of the total population). Most of them live in the four major cities ${ }^{(5)}$. The prevalence of overweight and obesity is high among Turkish, Moroccan and Surinamese migrants of the first generation (i.e. those who were born in the country of origin), in particular among women ${ }^{(6-13)}$. Possible underlying mechanisms are changes in lifestyle that result from the process of migration combined with the socio-cultural norms among non-Western migrants on food intake, 
physical activity patterns and perceptions regarding body weight ${ }^{(14)}$. However, it is not clear how overweight prevalence develops in their offspring (i.e. the second generation), while the number of young second-generation migrants from Turkey, Morocco and Surinam will form an increasing part of the Dutch population in the future ${ }^{(15)}$. We expect the overweight prevalence among secondgeneration migrants to converge to that of the host population since this generation has not been through the process of migration and will be more likely to adopt the attitudes and norms of the host population. Previously, a converging trend was shown among young secondgeneration Turkish and Moroccan women, but a diverging trend was found in second-generation Turkish men ${ }^{(16)}$. To our knowledge, no previous study on overweight development among second-generation Surinamese migrants has been done.

Our study investigates the prevalence of overweight among second-generation Turkish, Moroccan and Surinamese migrants aged 16-34 years in order to identify future risk groups. Our main research questions were:

1. Does the prevalence of overweight and obesity differ between second-generation Turkish, Moroccan and Surinamese migrants and their ethnic Dutch peers?

2. Does the prevalence of overweight and obesity differ between second- and first-generation migrants?

We also studied whether sociodemographic and socioeconomic factors could explain differences in overweight prevalence.

\section{Methods}

\section{Data collection and participants}

Data for the present study were collected as part of a general health questionnaire in 2008 in the four major cities of the Netherlands: Amsterdam, The Hague, Rotterdam and Utrecht. A sample of 42686 non-institutionalised residents aged 16 years and over was drawn from the municipal population registers. The total response rate was 49\% (Dutch: 57\%; Turkish: 41\%; Moroccan: $30 \%$; Surinamese: $40 \%$ ). Respondents filled out a questionnaire on paper (85\%) or on the Internet (15\%). A trained interviewer helped nine respondents $(0 \cdot 2 \%)$ to fill out the questionnaire. For our study we analysed data from second-generation Surinamese ( $n$ 230), Turkish ( $n$ 203) and Moroccan ( $n$ 172) respondents and compared them with their ethnic Dutch peers ( $n$ 3615). We also compared them with first-generation Surinamese ( $n$ 139), Turkish ( $n$ 241) and Moroccan ( $n$ 187) respondents. Analyses were limited to respondents aged 16-34 years in both the first and second generation, because of the low number of second-generation Turks, Moroccans and Surinamese over 35 years of age in the Netherlands ${ }^{(15)}$. Respondents without data on body weight and/or height were excluded from the analyses ( $n$ 137).
Participants received written information about the health survey. The study protocol was approved by the Medical Ethical Committee of the Academic Medical Centre, University of Amsterdam, the Netherlands.

\section{Measurements}

Overweight was defined as $\mathrm{BMI} \geq 25 \cdot 0 \mathrm{~kg} / \mathrm{m}^{2}$ and obesity as $\mathrm{BMI} \geq 30 \cdot 0 \mathrm{~kg} / \mathrm{m}^{2}$, with BMI calculated from selfreported weight and height. Demographic characteristics included sex, age and marital status (whether or not married or cohabiting). Socio-economic position included level of education completed (four levels: primary school or less; low vocational education; intermediate vocational education; high vocational education and university), financial situation (whether or not able to manage on the household income) and employment status (whether or not employed/student). Respondents who had not yet completed their education were categorised in this (uncompleted) level of education. Ethnic origin was based on the respondent's country of birth and the country of birth of the respondent's parents. Participants born in Turkey, Morocco or Surinam were classified as firstgeneration migrants, unless both parents were born in the Netherlands. Second-generation migrants were born in the Netherlands and had at least one parent born abroad.

\section{Statistical analysis}

First, means and prevalence rates of several background characteristics, overweight and obesity were calculated. Second, using a logistic regression model we tested differences in overweight and obesity of second-generation migrants $v$. the ethnic Dutch and first-generation migrants (model 1). Age was added in model 2 and educational level in model 3. The analyses were stratified for sex because of the large differences in overweight prevalence between men and women. We analysed data using the statistical software package SPSS version $17 \cdot 0$.

\section{Results}

\section{Sample characteristics}

Table 1 shows the sociodemographic characteristics as well as the prevalence of overweight and obesity among the study population. Second-generation migrant groups were on average younger and less often married or cohabiting compared with the ethnic Dutch and firstgeneration migrants. About a quarter of the secondgeneration migrants had a low education (level 1 and 2), whereas this applied to about one out of ten Dutch respondents and to $25-50 \%$ of the first generation. Unemployment and being a homemaker were more prevalent among second-generation migrants than among the Dutch. Highest numbers of unemployment and being a homemaker were found among Turks and Moroccans of the first generation. The prevalence of an unfavourable 
Table 1 Crude characteristics of the study population by sex and ethnic group; respondents (aged 16 years and over) to a cross-sectional postal and online health survey, four major Dutch cities, 2008

\begin{tabular}{|c|c|c|c|c|c|c|c|c|c|c|c|c|c|c|}
\hline & & & \multicolumn{4}{|c|}{ Surinamese } & \multicolumn{4}{|c|}{ Turkish } & \multicolumn{4}{|c|}{ Moroccan } \\
\hline & \multicolumn{2}{|c|}{ Dutch } & \multicolumn{2}{|c|}{ 1st generation } & \multicolumn{2}{|c|}{ 2nd generation } & \multicolumn{2}{|c|}{ 1st generation } & \multicolumn{2}{|c|}{ 2nd generation } & \multicolumn{2}{|c|}{ 1st generation } & \multicolumn{2}{|c|}{ 2nd generation } \\
\hline & $\begin{array}{l}\text { Mean } \\
\text { or \% }\end{array}$ & $\begin{array}{c}\text { SD or } \\
95 \% \mathrm{Cl}\end{array}$ & $\begin{array}{l}\text { Mean } \\
\text { or \% }\end{array}$ & $\begin{array}{c}\text { SD or } \\
95 \% \mathrm{Cl}\end{array}$ & $\begin{array}{l}\text { Mean } \\
\text { or \% }\end{array}$ & $\begin{array}{c}\text { SD or } \\
95 \% \mathrm{Cl}\end{array}$ & $\begin{array}{l}\text { Mean } \\
\text { or } \%\end{array}$ & $\begin{array}{c}\text { SD or } \\
95 \% \mathrm{Cl}\end{array}$ & $\begin{array}{l}\text { Mean } \\
\text { or \% }\end{array}$ & $\begin{array}{c}\text { SD or } \\
95 \% \mathrm{Cl}\end{array}$ & $\begin{array}{l}\text { Mean } \\
\text { or \% }\end{array}$ & $\begin{array}{c}\text { SD or } \\
95 \% \mathrm{Cl}\end{array}$ & $\begin{array}{l}\text { Mean } \\
\text { or \% }\end{array}$ & $\begin{array}{c}\text { SD or } \\
95 \% \mathrm{Cl}\end{array}$ \\
\hline Men & \multicolumn{2}{|c|}{$(n$ 1453) } & \multicolumn{2}{|c|}{$(n 55)$} & \multicolumn{2}{|c|}{$(n 75)$} & \multicolumn{2}{|c|}{$(n$ 115) } & \multicolumn{2}{|c|}{$(n 97)$} & \multicolumn{2}{|c|}{$(n 75)$} & \multicolumn{2}{|c|}{$(n 65)$} \\
\hline Age (mean; SD) & $25 \cdot 7$ & $5 \cdot 6$ & $26 \cdot 2$ & $6 \cdot 1$ & $22 \cdot 7$ & $5 \cdot 7$ & $27 \cdot 6$ & $5 \cdot 3$ & $20 \cdot 3$ & 4.5 & $27 \cdot 2$ & $6 \cdot 0$ & $20 \cdot 1$ & $4 \cdot 4$ \\
\hline Married or cohabiting (\%) & $40 \cdot 4$ & - & $37 \cdot 0$ & - & $13 \cdot 7$ & - & $73 \cdot 0$ & - & $17 \cdot 4$ & - & $55 \cdot 4$ & - & $12 \cdot 9$ & - \\
\hline \multicolumn{15}{|l|}{ Educational level (\%) } \\
\hline 2 & $9 \cdot 4$ & - & $28 \cdot 8$ & _- & $18 \cdot 1$ & _- & $30 \cdot 4$ & - & $\begin{array}{r}5 \cdot 6 \\
21 \cdot 3\end{array}$ & - & $\begin{array}{l}16 \cdot 0 \\
29 \cdot 3\end{array}$ & $\begin{array}{c}- \\
-\end{array}$ & $\begin{array}{r}3 \cdot 3 \\
24 \cdot 6\end{array}$ & $\begin{array}{l}- \\
-\end{array}$ \\
\hline 3 & $28 \cdot 1$ & - & $48 \cdot 1$ & - & $48 \cdot 6$ & - & $30 \cdot 4$ & - & $49 \cdot 4$ & - & 36.0 & - & $45 \cdot 9$ & - \\
\hline 4 (highest) & $59 \cdot 1$ & - & $21 \cdot 2$ & - & $26 \cdot 4$ & - & 23.5 & - & $23 \cdot 6$ & - & $18 \cdot 7$ & - & $26 \cdot 2$ & - \\
\hline Unfavourable financial situation (\%) & $17 \cdot 0$ & - & $29 \cdot 6$ & - & $32 \cdot 9$ & - & $61 \cdot 3$ & - & $23 \cdot 3$ & - & $41 \cdot 7$ & - & $32 \cdot 3$ & - \\
\hline \multicolumn{15}{|l|}{ Employment status (\%) } \\
\hline Paid employment & $70 \cdot 9$ & - & $77 \cdot 4$ & - & $49 \cdot 3$ & - & $75 \cdot 9$ & - & $39 \cdot 4$ & - & $72 \cdot 2$ & - & $45 \cdot 2$ & - \\
\hline Unemployed & $2 \cdot 4$ & - & $7 \cdot 5$ & - & $7 \cdot 0$ & - & $10 \cdot 2$ & - & $4 \cdot 3$ & - & $9 \cdot \overline{7}$ & - & $6 \cdot 5$ & - \\
\hline Homemaker & $0 \cdot 1$ & - & 0.0 & - & 0.0 & - & 0.0 & - & 0.0 & - & $1 \cdot 4$ & - & 0.0 & - \\
\hline Student & $26 \cdot 5$ & - & $15 \cdot 1$ & - & $43 \cdot 7$ & - & $13 \cdot 9$ & - & $56 \cdot 4$ & - & $16 \cdot 7$ & - & $48 \cdot 4$ & - \\
\hline \multicolumn{15}{|l|}{ Weight status } \\
\hline Overweight/obeset $(\% ; 95 \% \mathrm{Cl})$ & $24 \cdot 0$ & $21 \cdot 8,26 \cdot 2$ & $38 \cdot 2$ & $26 \cdot 4,51 \cdot 6$ & $44 \cdot 0$ & $33 \cdot 2,55 \cdot 4$ & $45 \cdot 2$ & $36 \cdot 4,54 \cdot 4$ & $39 \cdot 2$ & $30 \cdot 0,49 \cdot 2$ & $49 \cdot 3$ & $38 \cdot 2,60 \cdot 5$ & $16 \cdot 9$ & $9 \cdot 6,28 \cdot 0$ \\
\hline Obese‡ (\%; $95 \% \mathrm{Cl})$ & $4 \cdot 0$ & $3 \cdot 1,5 \cdot 1$ & $3 \cdot 6$ & $0 \cdot 9,13 \cdot 4$ & $8 \cdot 0$ & $3 \cdot 6,16 \cdot 7$ & $9 \cdot 6$ & $5 \cdot 4,16 \cdot 5$ & $2 \cdot 1$ & $0 \cdot 5,7 \cdot 9$ & $10 \cdot 7$ & $5 \cdot 4,19 \cdot 9$ & $3 \cdot 1$ & $0.8,11 \cdot 5$ \\
\hline \multicolumn{3}{|l|}{ Women } & \multicolumn{2}{|c|}{$(n 84)$} & \multicolumn{2}{|c|}{$(n$ 155) } & & 26) & & 06) & & 112) & & 07) \\
\hline Age (mean; sD) & $25 \cdot 4$ & $5 \cdot 4$ & $27 \cdot 1$ & $6 \cdot 2$ & $21 \cdot 3$ & 4.9 & $27 \cdot 6$ & $5 \cdot 1$ & $21 \cdot 3$ & $5 \cdot 4$ & $26 \cdot 7$ & $5 \cdot 3$ & $20 \cdot 9$ & $4 \cdot 7$ \\
\hline Married or cohabiting (\%) & $44 \cdot 8$ & - & $35 \cdot 4$ & - & $12 \cdot 7$ & - & $73 \cdot 0$ & - & $26 \cdot 0$ & - & $70 \cdot 0$ & - & $23 \cdot 1$ & - \\
\hline Years in the Netherlands (mean; SD) & NA & - & $17 \cdot 1$ & $9 \cdot 9$ & NA & - & $14 \cdot 1$ & $8 \cdot 8$ & NA & - & $15 \cdot 0$ & $8 \cdot 1$ & NA & - \\
\hline Educational level (\%) & & & & & & & & & & & & & & \\
\hline 1 (lowest) & $1 \cdot 3$ & - & $3 \cdot 7$ & - & $3 \cdot 4$ & - & $29 \cdot 5$ & - & $5 \cdot 0$ & - & $25 \cdot 2$ & - & $4 \cdot 0$ & - \\
\hline 2 & $8 \cdot 4$ & - & $22 \cdot 0$ & - & $15 \cdot 4$ & - & $20 \cdot 5$ & - & $20 \cdot 8$ & - & $25 \cdot 2$ & - & $18 \cdot 8$ & - \\
\hline 3 & $23 \cdot 9$ & - & $45 \cdot 1$ & - & $45 \cdot 6$ & - & $34 \cdot 4$ & - & $49 \cdot 5$ & - & $32 \cdot 0$ & - & $41 \cdot 6$ & - \\
\hline 4 (highest) & $66 \cdot 4$ & - & $29 \cdot 3$ & - & $35 \cdot 6$ & - & $15 \cdot 6$ & - & $24 \cdot 8$ & - & $17 \cdot 5$ & - & $35 \cdot 6$ & - \\
\hline Unfavourable financial situation (\%) & $21 \cdot 9$ & - & $42 \cdot 2$ & - & $28 \cdot 1$ & - & $53 \cdot 7$ & - & $37 \cdot 9$ & - & $38 \cdot 7$ & - & $33 \cdot 3$ & - \\
\hline Employment status (\%) & & & & & & & & & & & & & & \\
\hline Paid employment & $66 \cdot 6$ & - & $66 \cdot 3$ & - & $47 \cdot 4$ & - & $34 \cdot 4$ & - & $41 \cdot 2$ & - & $39 \cdot 0$ & - & $43 \cdot 7$ & - \\
\hline Unemployed & $2 \cdot 6$ & - & $6 \cdot 0$ & - & $7 \cdot 2$ & - & $13 \cdot 9$ & - & $8 \cdot 8$ & - & $15 \cdot 2$ & - & $6 \cdot 8$ & - \\
\hline Homemaker & $1 \cdot 6$ & - & $3 \cdot 6$ & - & $1 \cdot 3$ & - & $33 \cdot 6$ & - & $7 \cdot 8$ & - & $26 \cdot 7$ & - & $5 \cdot 8$ & - \\
\hline Student & $29 \cdot 2$ & - & $24 \cdot 1$ & - & $44 \cdot 1$ & - & $18 \cdot 0$ & - & $42 \cdot 2$ & - & $19 \cdot 0$ & - & $43 \cdot 7$ & - \\
\hline Weight status & & & & & & & & & & & & & & \\
\hline Overweight/obeset (\%; $95 \% \mathrm{Cl})$ & $16 \cdot 9$ & $15 \cdot 4,18 \cdot 6$ & $35 \cdot 7$ & $26 \cdot 2,46 \cdot 5$ & $23 \cdot 2$ & $17 \cdot 2,30 \cdot 5$ & $49 \cdot 2$ & $40 \cdot 6,57 \cdot 9$ & $21 \cdot 7$ & $14 \cdot 9,30 \cdot 6$ & $50 \cdot 0$ & $40 \cdot 8,59 \cdot 2$ & $26 \cdot 2$ & $18 \cdot 7,35 \cdot 3$ \\
\hline Obeseł $(\% ; 95 \% \mathrm{Cl})$ & $4 \cdot 1$ & $3 \cdot 4,5 \cdot 0$ & $6 \cdot 0$ & $2 \cdot 5,13.5$ & 9.0 & $5 \cdot 4,14 \cdot 7$ & $13 \cdot 5$ & $8 \cdot 6,20 \cdot 6$ & $12 \cdot 3$ & $7 \cdot 3,20 \cdot 0$ & $17 \cdot 0$ & $11 \cdot 1,25 \cdot 1$ & $13 \cdot 1$ & $7 \cdot 9,20 \cdot 9$ \\
\hline
\end{tabular}

NA, not applicable.

Overweigh/obese: $\mathrm{BMI} \geq 25 \cdot 0 \mathrm{~kg} / \mathrm{m}^{2}$

‡Obese: $\mathrm{BMI} \geq 30.0 \mathrm{~kg} / \mathrm{m}^{2}$. 
household financial situation varied from $17 \%$ in Dutch men to $61 \%$ in Turkish women of the first generation.

Among second-generation migrants, the prevalence of overweight (including obesity) was highest among Surinamese men (44\%) and Turkish men (39\%) and lowest in Moroccan men (17\%). Obesity rates varied from $2 \%$ among Turkish men to $13 \%$ among Moroccan women. In first-generation migrants, the prevalence of overweight ranged from $36 \%$ in Surinamese women to $50 \%$ in Moroccan women, while the obesity prevalence varied from $4 \%$ in Surinamese men to $17 \%$ in Moroccan women. Obesity was reported by $4 \%$ of the Dutch participants and overweight by $24 \%$ of Dutch men and $17 \%$ of Dutch women.

\section{Ethnic differences in overweight and obesity}

The combined overweight and obesity prevalence (hereafter referred to as 'overweight') in second-generation Turkish women and Moroccan men was similar to the Dutch figures. In second-generation Surinamese, Turkish men and Moroccan women, the prevalence of overweight was higher than among the Dutch (Table 2). The addition of age to the model increased the differences. Controlling for educational level attenuated ethnic differences in overweight. However, overweight was still more prevalent among second-generation Surinamese men and women, Turkish men and Moroccan women than among the Dutch. Table 2 also gives the results of the analyses for obesity only (BMI $\geq 30 \cdot 0 \mathrm{~kg} / \mathrm{m}^{2}$ ). No differences in obesity prevalence were found for secondgeneration migrant men aged 16-34 years compared with Dutch men. Migrant women of the second generation were more often obese than Dutch women. Controlling for age increased the ethnic differences in obesity among women, while these were reduced by adding level of education to the model. In both men and women, there was no significant interaction between educational level and ethnicity. This means that in all ethnic groups the lowest educated were at highest risk of being overweight or obese.

We also studied differences in overweight between second- and first-generation migrants. In all subgroups, except for Surinamese men, second-generation migrants seemed to have lower levels of overweight than the first generation (Table 3). After controlling for age, differences between the second and first generation were statistically significant only among Surinamese $(\mathrm{OR}=2 \cdot 48$; $95 \% \mathrm{CI}$ $1 \cdot 06,5 \cdot 83)$ and Moroccan men $(\mathrm{OR}=0 \cdot 33 ; 95 \%$ CI $0 \cdot 13$, $0 \cdot 83)$. Obesity prevalence in second-generation migrants did not differ from first-generation migrants, except for second-generation Turkish men who less often reported obesity than first generation (Table 3). After controlling for age, differences in obesity between the second- and first-generation Surinamese women were statistically significant. In general, adding educational level to the model did not influence the differences in overweight and obesity between second- and first-generation migrants. Only the difference in obesity prevalence between second- and first-generation Turkish women increased after controlling for educational level.

\section{Discussion}

We showed that after controlling for age, overweight is more prevalent in most young second-generation nonWestern migrant groups in comparison to the Dutch population, except for Moroccan men. Obesity rates among second-generation non-Western migrant young Turkish and Moroccan men were similar to those among the Dutch. However, second-generation non-Western migrant young women and Surinamese men were more often obese than their Dutch peers. Differences in the overweight prevalence between second- and first-generation non-Western migrants were present only among Surinamese and Moroccan men, with a higher overweight prevalence in second-generation Surinamese men and a lower prevalence in second-generation Moroccan men compared with the first generation. The higher prevalence of overweight and obesity among second-generation migrants compared with the ethnic Dutch was partly explained by their lower level of education.

As we found a higher overweight prevalence in most second-generation Turkish, Moroccan and Surinamese migrant subgroups compared with the ethnic Dutch population and a similar prevalence as among the first generation, our study does not support the hypothesis of convergence of the overweight prevalence of secondgeneration migrants towards the host population. Apparently, the overweight differences between secondand first-generation migrants in the Netherlands are more diverse than previously found ${ }^{(16)}$. This corresponds to earlier studies in the USA that have shown a variable association between acculturation and BMI depending on ethnic background and gender ${ }^{(17-20)}$. A possible explanation for the high overweight levels among secondgeneration migrants may lie in differences in lifestyle, including diet and physical activity, as well as other sociocultural factors. Migrant groups in the Netherlands place great value in their traditional food cultures. Given an environment of abundance, this may lead to overeating and overweight development ${ }^{(21)}$. The influence of age and educational level on overweight in migrants was found earlier. In contrast to earlier studies, marital status, financial situation and employment status did not add to the explanation of ethnic differences in overweight (results not presented $)^{(9,13,16)}$.

\section{Strengtbs and limitations}

Our study offered a new comparison of young secondgeneration migrant groups, giving additional insight into ethnic health inequalities. We had the unique opportunity to study data on overweight among a large sample of second-generation non-Western migrant groups from the 
Table 2 Differences in overweight and obesity prevalence of 16-34-year-old second-generation migrants $v$. ethnic Dutch, four major Dutch cities, 2008 (odds ratios and $95 \%$ confidence intervals)

\begin{tabular}{|c|c|c|c|c|c|c|c|c|c|c|c|c|}
\hline & \multicolumn{4}{|c|}{ Surinamese } & \multicolumn{4}{|c|}{ Turkish } & \multicolumn{4}{|c|}{ Moroccan } \\
\hline & \multicolumn{2}{|r|}{ Men } & \multicolumn{2}{|r|}{ Women } & \multicolumn{2}{|r|}{ Men } & \multicolumn{2}{|r|}{ Women } & \multicolumn{2}{|r|}{ Men } & \multicolumn{2}{|r|}{ Women } \\
\hline & OR & $95 \% \mathrm{Cl}$ & OR & $95 \% \mathrm{Cl}$ & OR & $95 \% \mathrm{Cl}$ & OR & $95 \% \mathrm{Cl}$ & OR & $95 \% \mathrm{Cl}$ & OR & $95 \% \mathrm{Cl}$ \\
\hline \multicolumn{13}{|c|}{ Overweight $\left(\mathrm{BMI} \geq 25 \cdot 0 \mathrm{~kg} / \mathrm{m}^{2}\right)$} \\
\hline \multicolumn{13}{|l|}{ Model 1} \\
\hline 2nd generation & $2 \cdot 50$ & $1 \cdot 56,4 \cdot 00^{\star * *}$ & 1.49 & $1 \cdot 01,2 \cdot 19^{*}$ & $2 \cdot 05$ & $1 \cdot 34,3 \cdot 13^{\star *}$ & $1 \cdot 36$ & $0 \cdot 85,2 \cdot 19$ & 0.65 & $0.33,1 \cdot 25$ & $1 \cdot 74$ & $1 \cdot 11,2 \cdot 72^{*}$ \\
\hline Dutch & $1 \cdot 00$ & Ref. & 1.00 & Ref. & 1.00 & Ref. & 1.00 & Ref. & 1.00 & Ref. & $1 \cdot 00$ & Ref. \\
\hline \multicolumn{13}{|l|}{ Model 2: + age } \\
\hline 2nd generation & $3 \cdot 72$ & $2 \cdot 25,6 \cdot 14^{\star \star *}$ & 1.95 & $1 \cdot 30,2 \cdot 92^{\star \star}$ & 3.93 & $2 \cdot 47,6 \cdot 25^{\star \star *}$ & $1 \cdot 78$ & $1 \cdot 09,2 \cdot 90^{\star}$ & $1 \cdot 20$ & $0 \cdot 60,2 \cdot 38$ & $2 \cdot 36$ & $1 \cdot 49,3 \cdot 74^{\star \star \star}$ \\
\hline Dutch & $1 \cdot 00$ & Ref. & 1.00 & Ref. & 1.00 & Ref. & 1.00 & Ref. & 1.00 & Ref. & 1.00 & Ref. \\
\hline \multicolumn{13}{|c|}{ Model 3: + age + educational level } \\
\hline 2nd generation & $3 \cdot 28$ & $1 \cdot 95,5 \cdot 53^{\star \star \star}$ & $1 \cdot 68$ & $1 \cdot 11,2 \cdot 53^{*}$ & $3 \cdot 43$ & $2 \cdot 11,5 \cdot 57^{\star \star \star}$ & $1 \cdot 29$ & $0 \cdot 77,2 \cdot 16$ & 0.94 & $0 \cdot 46,1 \cdot 95$ & $1 \cdot 97$ & $1 \cdot 22,3 \cdot 19^{\star \star}$ \\
\hline Dutch & $1 \cdot 00$ & Ref. & $1 \cdot 00$ & Ref. & $1 \cdot 00$ & Ref. & $1 \cdot 00$ & Ref. & $1 \cdot 00$ & Ref. & $1 \cdot 00$ & Ref. \\
\hline \multirow{2}{*}{\multicolumn{13}{|c|}{ Obesity $\left(\mathrm{BMI} \geq 30.0 \mathrm{~kg} / \mathrm{m}^{2}\right)$}} \\
\hline & & & & & & & & & & & & \\
\hline 2nd generation & $2 \cdot 09$ & $0 \cdot 87,5 \cdot 02$ & $2 \cdot 31$ & $1 \cdot 28,4 \cdot 17^{\star *}$ & 0.51 & $0 \cdot 12,2 \cdot 11$ & $3 \cdot 26$ & $1 \cdot 76,6 \cdot 04^{\star * *}$ & 0.76 & $0 \cdot 18,3 \cdot 20$ & $3 \cdot 51$ & $1 \cdot 92,6 \cdot 39^{\star \star \star}$ \\
\hline Dutch & $1 \cdot 00$ & Ref. & $1 \cdot 00$ & Ref. & $1 \cdot 00$ & Ref. & $1 \cdot 00$ & Ref. & $1 \cdot 00$ & Ref. & $1 \cdot 00$ & Ref. \\
\hline \multicolumn{13}{|l|}{ Model 2: + age } \\
\hline 2nd generation & $2 \cdot 80$ & $1 \cdot 14,6 \cdot 87^{\star}$ & $3 \cdot 44$ & $1 \cdot 86,6 \cdot 37^{\star \star \star}$ & $0 \cdot 88$ & $0 \cdot 21,3 \cdot 78$ & $4 \cdot 84$ & $2 \cdot 53,9 \cdot 23^{\star \star *}$ & $1 \cdot 37$ & $0 \cdot 31,5 \cdot 93$ & $5 \cdot 53$ & $2 \cdot 93,10 \cdot 4^{\star \star \star}$ \\
\hline Dutch & $1 \cdot 00$ & Ref. & $1 \cdot 00$ & Ref. & $1 \cdot 00$ & Ref. & $1 \cdot 00$ & Ref. & $1 \cdot 00$ & Ref. & $1 \cdot 00$ & Ref. \\
\hline \multicolumn{13}{|c|}{ Model 3: + age + educational level } \\
\hline 2nd generation & 1.94 & $0 \cdot 77,4 \cdot 89$ & $2 \cdot 85$ & $1 \cdot 51,5 \cdot 36^{\star \star}$ & 0.56 & $0 \cdot 13,2 \cdot 44$ & $3 \cdot 11$ & $1 \cdot 56,6 \cdot 20^{\star \star}$ & 0.92 & $0.21,4.05$ & $4 \cdot 71$ & $2 \cdot 44,9 \cdot 10^{\star \star \star}$ \\
\hline Dutch & $1 \cdot 00$ & Ref. & $1 \cdot 00$ & Ref. & $1 \cdot 00$ & Ref. & $1 \cdot 00$ & Ref. & $1 \cdot 00$ & Ref. & $1 \cdot 00$ & Ref. \\
\hline
\end{tabular}

Ref., referent category.

${ }^{\star} P<0.05,{ }^{* \star} P<0.01,{ }^{* \star *} P<0.000$ 
Table 3 Differences in overweight and obesity prevalence of 16-34-year-old second-generation migrants $v$. first-generation migrants, four major Dutch cities, 2008 (odds ratios and $95 \%$ confidence intervals)

\begin{tabular}{|c|c|c|c|c|c|c|c|c|c|c|c|c|}
\hline & \multicolumn{4}{|c|}{ Surinamese } & \multicolumn{4}{|c|}{ Turkish } & \multicolumn{4}{|c|}{ Moroccan } \\
\hline & \multicolumn{2}{|r|}{ Men } & \multicolumn{2}{|c|}{ Women } & \multicolumn{2}{|r|}{ Men } & \multicolumn{2}{|r|}{ Women } & \multicolumn{2}{|r|}{ Men } & \multicolumn{2}{|c|}{ Women } \\
\hline & OR & $95 \% \mathrm{Cl}$ & OR & $95 \% \mathrm{Cl}$ & OR & $95 \% \mathrm{Cl}$ & OR & $95 \% \mathrm{Cl}$ & OR & $95 \% \mathrm{Cl}$ & OR & $95 \% \mathrm{Cl}$ \\
\hline \multicolumn{13}{|c|}{ Overweight $\left(\mathrm{BMI} \geq 25 \cdot 0 \mathrm{~kg} / \mathrm{m}^{2}\right)$} \\
\hline \multicolumn{13}{|c|}{ Model 1} \\
\hline 1st generation & 1.00 & $\begin{array}{c}0.63,2.59 \\
\text { Ref. }\end{array}$ & $\begin{array}{l}0.55 \\
1.00\end{array}$ & $\begin{array}{l}0.30,0.97^{\star} \\
\text { Ref. }\end{array}$ & $\begin{array}{l}0.78 \\
1.00\end{array}$ & $\begin{array}{c}0.45,1.35 \\
\text { Ref. }\end{array}$ & $\begin{array}{l}0.29 \\
1.00\end{array}$ & $\begin{array}{c}0.16,0.5 \\
\text { Ref. }\end{array}$ & $\begin{array}{l}0.21 \\
1.00\end{array}$ & $\begin{array}{c}0 \cdot 10,0.4 \\
\text { Ref. }\end{array}$ & $\begin{array}{l}0.35 \\
1.00\end{array}$ &  \\
\hline \multicolumn{13}{|l|}{ Model 2: + age } \\
\hline 2nd generation & $2 \cdot 48$ & $1 \cdot 06,5 \cdot 83^{*}$ & $1 \cdot 14$ & $0 \cdot 56,2 \cdot 29$ & $1 \cdot 60$ & $0 \cdot 78,3 \cdot 27$ & 0.56 & $0.29,1.08$ & 0.33 & $0 \cdot 13,0 \cdot 83^{\star}$ & 0.62 & $0 \cdot 32,1 \cdot 18$ \\
\hline 1st generation & $1 \cdot 00$ & Ref. & $1 \cdot 00$ & Ref. & $1 \cdot 00$ & Ref. & $1 \cdot 00$ & Ref. & $1 \cdot 00$ & Ref. & $1 \cdot 00$ & Ref. \\
\hline \multicolumn{13}{|c|}{ Model 3: + age + educational level } \\
\hline $\begin{array}{l}\text { 2nd generation } \\
\text { 1st generation }\end{array}$ & $\begin{array}{l}2 \cdot 88 \\
1 \cdot 00\end{array}$ & $\begin{array}{c}1 \cdot 16,7 \cdot 11^{*} \\
\text { Ref }\end{array}$ & $\begin{array}{l}1.06 \\
1.00\end{array}$ & $0 \cdot 52,2 \cdot 16$ & $\begin{array}{l}1.63 \\
1.00\end{array}$ & $0 \cdot 77,3 \cdot 43$ & $0 \cdot 64$ & $0 \cdot 32,1 \cdot 29$ & $0 \cdot 34$ & $0 \cdot 13,0 \cdot 88^{*}$ & $0 \cdot 73$ & $\begin{array}{c}0 \cdot 36,1 \cdot 50 \\
\text { Ref }\end{array}$ \\
\hline \multirow{2}{*}{\multicolumn{13}{|c|}{$\begin{array}{l}\text { Obesity }\left(\mathrm{BMI} \geq 30 \cdot 0 \mathrm{~kg} / \mathrm{m}^{2}\right) \\
\text { Model } 1\end{array}$}} \\
\hline & & & & & & & & & & & & \\
\hline 2nd generation & $2 \cdot 30$ & $0.45,11 \cdot 9$ & $1 \cdot 57$ & $0.55,4.52$ & $0 \cdot 20$ & $0.04,0.92^{\star}$ & 0.90 & $0.41,1.94$ & 0.27 & $0 \cdot 05,1 \cdot 30$ & 0.74 & $0.35,1.56$ \\
\hline 1st generation & $1 \cdot 00$ & Ref. & $1 \cdot 00$ & Ref. & $1 \cdot 00$ & Ref. & $1 \cdot 00$ & Ref. & $1 \cdot 00$ & Ref. & $1 \cdot 00$ & Ref. \\
\hline \multicolumn{13}{|l|}{ Model 2: + age } \\
\hline 2nd generation & $2 \cdot 29$ & $0 \cdot 42,12 \cdot 5$ & 3.46 & $1 \cdot 03,11 \cdot 7^{*}$ & 0.38 & $0 \cdot 07,2 \cdot 24$ & $2 \cdot 35$ & $0.94,5 \cdot 91$ & 0.43 & $0.07,2 \cdot 77$ & $1 \cdot 18$ & $0 \cdot 49,2 \cdot 83$ \\
\hline 1st generation & $1 \cdot 00$ & Ref. & $1 \cdot 00$ & Ref. & $1 \cdot 00$ & Ref. & $1 \cdot 00$ & Ref. & $1 \cdot 00$ & Ref. & 1.00 & Ref. \\
\hline \multicolumn{13}{|c|}{ Model 3: + age + educational level } \\
\hline 2nd generation & $2 \cdot 40$ & $0.43,13 \cdot 4$ & 3.44 & $0.99,11.9$ & 0.42 & $0.07,2 \cdot 55$ & $5 \cdot 42$ & $1 \cdot 71,17 \cdot 2^{\star *}$ & 0.50 & $0.08,3.40$ & 1.94 & $0 \cdot 70,5 \cdot 36$ \\
\hline 1st generation & 1.00 & Ref. & $1 \cdot 00$ & Ref. & $1 \cdot 00$ & Ref. & $1 \cdot 00$ & Ref. & 1.00 & Ref. & 1.00 & Ref. \\
\hline
\end{tabular}

Ref., referent category.
${ }^{\star} P<0.05,{ }^{*} P<0.01,{ }^{* * *} P<0.000$ 
four major cities in the Netherlands. In particular, data on overweight among young second-generation Surinamese have not previously been studied. The study has some limitations. First, data collection resulted in a fairly low response rate of 50\%, which was lower among 16-34year-old migrants (33\%). This might negatively influence the validity of our study, although large-scale survey research among the migrant population in the Netherlands usually results in response rates of this value. Nevertheless, selection bias might have influenced the external validity. Second, BMI was based on self-reports which often results in an underestimation of body weight and an overestimation of body height. Consequently, the true prevalence of overweight in our study population was presumably higher than shown herein. Some previous studies have shown ethnic differences in underestimation of body weight and height ${ }^{(12,22-24)}$. For example, it was shown that the accuracy of self-reports was smaller among Turkish and Moroccan women than among Dutch women. In Turkish and Moroccan men, no ethnic differences in underestimation of body weight and height were found, while in the Surinamese discrepancies between measurements and self-reports were smaller than in the Dutch. Therefore, the true differences in overweight between second-generation migrants and the ethnic Dutch may be larger among Turkish and Moroccan women and smaller among Surinamese men and women than reported in our study. Third, our results might not apply to all individuals within a migrant group, because of heterogeneity within the group. In particular the Surinamese group consists of a mix of ethnicities with $36 \%$ being Hindustani-Surinamese (originally from the Indian subcontinent), $41 \%$ AfricanSurinamese (predominantly of African origin) and 23\% having another or mixed background ${ }^{(25)}$. In our data set we could not differentiate between these Surinamese subgroups, while a previous study demonstrated differences in overweight prevalence between African-Surinamese and South Asian-Surinamese women ${ }^{(26)}$. Finally, the educational level of young people may not reflect their socio-economic position, as they might not have finished their education yet. For those people who were still following a course of study we augmented the highest level of education attained. Our assumption that this would be their final education level may nevertheless be false, which might have resulted in an over-representation of higher educational levels.

\section{Implications and conclusions}

The high prevalence of overweight and obesity in migrant origin groups poses a major risk for chronic diseases, including type 2 diabetes, hypertension, CVD and cancer $^{(1)}$. Not only first-generation migrants are at risk, but also most second-generation migrant groups have higher overweight and obesity rates than the ethnic Dutch. Our study did not show a converging trend for overweight among second-generation migrants towards the Dutch host population. In all groups, overweight and obesity levels rise strongly with increasing age and lower educated groups are at higher risk of having overweight and obesity. Public health policies aimed at reducing overweight should therefore not ignore second-generation migrants, particularly those with a lower education level. Cultural factors and barriers should be considered when developing overweight prevention programmes for migrant groups, e.g. the fact that among migrant groups food plays a central role in expressing hospitality, which may result in a social context where food is abundant and difficult to refuse ${ }^{(21)}$.

\section{Acknowledgements}

Sources of funding: This work was supported by the Municipality of Amsterdam, the Public Health Service of Amsterdam, the Municipality of Rotterdam, the Municipality of The Hague, the Public Health Service of The Hague and the Municipality of Utrecht. The Municipality of Amsterdam, the Public Health Service of Amsterdam, the Municipality of Rotterdam, the Municipality of The Hague, the Public Health Service of The Hague and the Municipality of Utrecht had no role in the design, analysis or writing of this article. Conflicts of interest: There are no conflicts of interest to declare. Authors' contributions: H.D. was the main researcher. H.D., J.K.U.-V., A.J.B.-N., G.M.S. and M.P.H.B. designed the health survey and coordinated the data collection. All authors contributed to the interpretation of results. H.D., M.N. and J.K.U.-V. drafted the manuscript and all authors critically reviewed it for scientific content and approved the final version.

\section{References}

1. World Health Organization (2000) Obesity: Preventing and Managing the Global Epidemic. Report of a WHO Consultation. WHO Technical Report Series no. 894. Geneva: WHO.

2. Gast GC, Frenken FJ, van Leest LA et al. (2007) Intranational variation in trends in overweight and leisure time physical activities in The Netherlands since 1980: stratification according to sex, age and urbanisation degree. Int $J$ Obes (Lond) 31, 515-520.

3. Salmond CE, Prior IAM \& Wessen AF (1989) Blood pressure patterns and migration: a 14-year cohort study of adult Tokelauans. Am J Epidemiol 130, 37-52.

4. Sundquist J \& Johansson SE (1998) The influence of socioeconomic status, ethnicity and lifestyle on body mass index in a longitudinal study. Int J Epidemiol 27, 57-63.

5. Nicolaas H, Wobma E \& Ooijevaar J (2010) Demografie (Demography). In Jaarrapport Integratie (Annual Report Integration), pp. 33-58 [R Van der Vliet, J Ooijevaar and A Boerdam, editors]. Den Haag: Centraal Bureau voor de Statistiek.

6. Brussaard JH, van Erp-Baart AM, Brants HA et al. (2001) Nutrition and health among migrants in The Netherlands. Public Health Nutr 4, 659-664.

7. Wändell PE, Ponzer S, Johansson SE et al. (2004) Country of birth and body mass index: a national study of 2,000 immigrants in Sweden. Eur J Epidemiol 19, 1005-1010. 
8. Kumar BN, Meyer HE, Wandel M et al. (2006) Ethnic differences in obesity among immigrants from developing countries, in Oslo, Norway. Int J Obes (Lond) 30, 684-690.

9. Cornelisse-Vermaat JR \& Maassen van den Brink HM (2007) Ethnic differences in lifestyle and overweight in the Netherlands. Obesity (Silver Spring) 15, 483-493.

10. Dijkshoorn H, Nierkens V \& Nicolaou M (2008) Risk groups for overweight and obesity among Turkish and Moroccan migrants in The Netherlands. Public Health 122, 625-630.

11. Gualdi-Russo E, Zironi A, Dallari GV et al. (2009) Migration and health in Italy: a multiethnic adult sample. J Travel Med 16, 88-95.

12. Valkengoed IGM, Nicolaou M \& Stronks K (2011) Ethnic differences in discrepancies between self-reported and measured weight, height and body mass index. Eur $J$ Public Health 21, 420-423.

13. Ujcic-Voortman JK, Bos G, Baan CA et al. (2011) Obesity and body fat distribution: ethnic and socioeconomic differences between Turkish, Moroccan and Dutch ethnic groups. Obesity Facts 4, 53-60.

14. Nicolaou M, Benjelloun S, Stronks K et al. (2012) Influences on body weight of female Moroccan migrants in the Netherlands: a qualitative study. Health Place 18, 883-891.

15. Statistics Netherlands (2012) Bevolking; generatie, geslacht, leeftijd en herkomstgroepering, 1 januari (Population; generation, sex, age and origin, January 1st). http:// statline.cbs.nl/StatWeb/publication/ (accessed January 2012).

16. Hosper K, Nierkens V, Nicolaou M et al. (2007) Behavioural risk factors in two generations of non-Western migrants: do trends converge towards the host population? Eur J Epidemiol 22, 163-172.

17. Hazuda HP, Haffner SM, Stern MP et al. (1988) Effects of acculturation and socioeconomic status on obesity and diabetes in Mexican Americans: the San Antonio Heart Study. Am J Epidemiol 128, 1289-1301.
18. Khan LK, Sobal J \& Martorell R (1997) Acculturation, socioeconomic status, and obesity in Mexican Americans, Cuban Americans, and Puerto Ricans. Int J Obes Relat Metab Disord 21, 91-96.

19. Sundquist J \& Winkleby M (2000) Country of birth, acculturation status and abdominal obesity in a national sample of Mexican-American women and men. Int $J$ Epidemiol 29, 470-477.

20. Sanghavi Goel M, McCarthy EP, Philips RS et al. (2004) Obesity among US immigrant subgroups by duration of residence. JAMA 292, 2860-2867.

21. Nicolaou M, Doak CM, Dam RM et al. (2009) Cultural and social influences on food consumption in Dutch residents of Turkish and Moroccan origin: a qualitative study. J Nutr Educ Behav 41, 232-241.

22. Gillum RF \& Sempos CT (2005) Ethnic variation in validity of classification of overweight and obesity using selfreported weight and height in American women and men: the Third National Health and Nutrition Examination Survey. Nutr J $\mathbf{4}, 27$.

23. Johnson WD, Bouchard C, Newton RL Jr et al. (2009) Ethnic differences in self-reported and measured obesity. Obesity (Silver Spring) 17, 571-577.

24. Dijkshoorn H, Ujcic-Voortman JK, Viet L et al. (2011) Ethnic variation in validity of the estimated obesity prevalence using self-reported weight and height measurements. $B M C$ Public Health 11, 408.

25. Choenni C \& Harmsen C (2007) Geboorteplaats en etnische samenstelling van Surinamers in Nederland (Place of birth and ethnic composition of the Surinamese in the Netherlands). Bevolkingstrends 1, 74-78.

26. Nicolaou M, van Valkengoed IG, Doak CM et al. (2012) Ethnic differences in self-rated overweight and association with reporting weight loss action: the SUNSET study. Eur J Public Health 22, 859-863. 